$\xi=-1$

\title{
Review on Upcoming Innovative Technology in Big Data
}

\author{
M. Therasa *, S. M. Poonkuzhali, M., Santhana Joyce, S. Annie Shery \\ ${ }^{1}$ Assoc. Professor, Department of Computer Science, Panimalar Institute of Technology, Chennai-600123. \\ *Corresponding author E-mail: mtth@gmail.com
}

\begin{abstract}
This paper is a survey of the big data idea, its measurements, its design correlation between the prior idea and the most recent, the capacity conceivable i.e. the databases and starting point of enormous information. The social database, having unbending pattern, has been winning since quite a while yet it is hard to store the unstructured information in social database. The unstructured information has principally message nature or is as logs. Here comes the idea of No SQL databases. Enormous Data is little information with huge information estimate.
\end{abstract}

Keywords: big data, dimensions, inconsistency, granularity.

\section{Introduction}

In the early years of 21 st century, by sensibility of the rising use of web, the term „Big Data" was showed up however out of the blue it got an impact everything pondered perfect around 2013, in light of some need. This need was the examination of data. Inspiration driving constrainment was never an issue. It was the disappointment of standard social databases that impacted the distinction in No SQL databases. The standard databases have firm case while the No SQL databases have versatile system without downtime (a condition when structure fails to perform noteworthy activities). It was then this data got unmistakable as monstrous data. Tremendous Data is on an exceptionally fundamental level little data with wide data measure. The data association gadgets which are accessible since decades imagine that it's difficult to plan scattered enlightening blends. Certain masterminding applications are in like way not set up to process such voluminous and dynamic data. Such enlightening records shape the immense data.

The cross of modernized data in 2011 was around 1.8 Zettabytes (1.8 trillion gigabytes) i.e. supporting structures association establishment needs to empower 50 times more information by year 2020. In like path, examinations of inspiration driving constrainment, money related issues and certification should be dealt with completely while including new immense data joining ruins with existing data and structures interest establishment [1]. After the outline of various outcomes of enormous data, the one appeared in [3] finally gives the most sensible significance of epic data i.e. data that is liberally goliath or a critical measure of vivacious or too hard for

at show known instruments to organize. Here, "to a great degree monstrous" makes a translation of that affiliations should engineer affecting petabyte-to scale get-togethers of data that started from snap streams, exchange histories, sensors, and elsewhere. "Staggeringly sharp" finds that is data epic, and in addition it must be encouraged quickly - for example, to perform misleading presentation at a condition of offer or handle which advance to show to a customer on a page. "Too hard" is a catchall for data that doesn't fit impeccably into a current getting ready contraption or that needs some kind of examination that present instruments can't rapidly give. Goliath Data is spreading inconceivably in the business. Most by a wide edge of the endeavors require the records of the work they do and what's more are tense to know the encapsulation of the buyer. This will lead business advantage. Epic Data is seeing the chance to be unmistakably with respect to all parts of human progression from essentially recording events to get some data about, structure, age and modernized affiliations or things progress to the last purchaser. The data made utilizing specific sources has arranged lead, qualities and nature. It contains certain objective and unessential information however an essential segment of the data is in printed plot. It is just the unstructured data.

\section{Foundation}

In the IT business everything considered, the lively move of Big Data has made new issues and issues concerning data affiliation and examination. Five standard issues are volume, blend, speed, regard, and multifaceted nature. In this audit, there are additional issues related to data, for instance, the smart difference in volume, blend,

regard, association, and security. Each issue looks out for a key issue of specific research that requires exchange. In like way, this examination proposes a data life cycle that uses the sorts of advance and wordings of Big Data. Future research presentation in this field are settled in light of shots and a couple of open issues in Big Data control.

\subsection{Volume of Big Data:}

The volume of Big Data is everything seen as wide. Regardless, it doesn't require a particular measure of petabytes. The change in the volume of various data records is however much of the time as could reasonably be expected coordinated by getting additional web gathering; in any case, the relative estimation of each datum strengthen diminishes in degree toward perspectives, for instance, age, sort, entire, and liberality. Along these lines, such utilize is 
insane. The running with two subsections detail the volume of Big Data in association with the sharp ability in data and the change rate of hard plate drives (HDDs). It what's more looks Data in the current state of tries and advances.

\subsubsection{Rapid Growth of Data:}

The data sort that augmentations most rapidly are unstructured data. This data oversee is delineated by "human information, for instance, top quality records, films, photos, stunning 'ol kept incitations, cash related trades, phone records, genomic datasets, seismic pictures, geospatial maps, email, tweets, Facebook data, call-center talks, remote calls, site clicks, reports, sensor data, telemetry, obliging records and pictures, climatology and air records, log accounts, and substance. As appeared by Computer World, unstructured information may disregard on $70 \%$ to $80 \%$ of all data in affiliations.

\subsubsection{Change Rate of Hard Disk Drives (HDDs):}

The criticalness for electronic securing is astoundingly versatile. It can't be completely met and is controlled just by spending frameworks and collusion most far away point and cutoff. tapes and circles and optical, solid state, and electromechanical contraptions. Before the electronic change, information was dominatingly secured in clear tapes as demonstrated by the open bits. Beginning at 2007, regardless, most data are secured in HDDs (52\%), trailed by optical social gathering (28\%) and affected tapes (around 11\%) Paper-based most far away point has dwindled $0.33 \%$ of each 1986 to $0.007 \%$ out of 2007 , paying little regard to the way that its capacity has perseveringly developed.

\section{Enormous DATA MANAGEMENT}

The working of Big Data must be synchronized with the sustain strategy of the affiliation. To date, most by a wide margin of the information utilized by affiliations are stale. Information is progressively sourced from different fields that are disillusioned and scattered, for example, data from machines or sensors and huge wellsprings of open and private information. Early, most affiliations were not skilled either catch or store these information, and accessible contraptions couldn't deal with the information in a sensible measure of time. In any case, the new Big Data change refreshes execution, strengthens progress in the things and relationship of plans of advancement, and gives basic activity brace Colossal Data advance plans to restrict mechanical get together and supervising costs and to attest the estimation of Big Data beforehand giving fundamental affiliation assets. Extremely sorted out Big Data are open, solid, secure, and sensible. Along these lines, Big Data applications can be associated in different complex insightful controls (either single or interdisciplinary), including air science, stargazing, cure, science, genomics, and biogeochemistry. In the running with a domain, we quickly overview information association contraptions and propose another information life cycle that uses the sorts of progress and wordings of Big Data.

\subsection{HADOOP:}

Hadoop is made in Java and is a best level Apache expand that began in 2006. It stresses introduction from the point of view of flexibility and examination to see close endless accomplishments. Doug Cutting made Hadoop as a social affair of open-source extends on which the Google Map Reduce programming condition could be associated in a spouted framework. Finally, it is utilized on immense wholes of information. With Hadoop, tries can oversee information that was by then difficult to coordinate and look at. Hadoop is utilized by around $63 \%$ of relationship to encourage goliath number of unstructured logs and occasions.

\subsection{HDFS:}

This point of view is associated when the measure of information is pointlessly for a solitary machine. HDFS is more character boggling than other record structures given the complexities and perils of systems. Pack contains two sorts of center interests. The administer focus point is a name-focus that goes about as a specialist focus. The second fixation point sort is a server develop point that goes about as slave focus. This kind of focus point comes in things. Near to these two sorts of focuses, HDFS can besides have relate name-focus point. HDFS stores records in keeps, the default piece size of which is $64 \mathrm{MB}$. All HDFS records are duplicated in things to enable the parallel planning of a lot of information.

\subsection{HBase:}

HBase is an alliance structure that is open-source, kept, and passed on in light of the BigTable of Google. This framework is part instead of push based, which accel-erates the execution of exercises over inside and out that truly matters ill-defined respects transversely completed sweeping enlightening get-togethers. For instance, read and impact attempts to join all lines however just a little subset of all fragments. HBase is open through application programming between faces (APIs, for example, Thrift, Java, and honest to goodness state exchange (REST). These APIs don't have their own particular demand or scripting tongues. As is typically done, HBase depends absolutely on a ZooKeeper occasion.

\subsection{ZooKeeper}

ZooKeeper coordinates to, blueprints, and names a critical measure of data. It additionally gives appropriated synchro-nization and get-together affiliations. This event attracts dis framework to direct and add to each other through a name space of data registers ( - centers) that is shared and particular leveled, for instance, a record structure. Alone, ZooKeeper is an appropriated advantage that contains star and slave center obsessions and stores structure information.tributed

\subsection{HCatalog}

HCatalog oversees HDFS. It stores metadata and produces tables for a great deal of data. HCatalog depends on Hive metastore and obliges it with various affiliations, including MapReduce and Pig, using a common data appear. With this data show up, HCatalog can in like path make to HBase. HCatalog streamlines customer correspondence using HDFS data and is a wellspring of data sharing among instruments and execution stages.

\section{Constraints OF HADOOP}

With Hadoop, inconceivably wide volumes of data with either detaching structures or none at all can be sorted out, created, and separated. In any case, Hadoop in like way has a few obstructions.

The Generation of Multiple Copies of Big Data: HDFS was worked for advantage; thusly, data is emphasized in things. Everything considered, data are made in triplicate at any rate. In any case, six copies must be abandoned on to keep execution through data space. As requirements be, the Big Data is made further.

Testing Framework: The Map Reduce structure is scattered, particularly when complex transformational legitimization must be used. Tries have been made by open-source modules to loosen up this structure, yet these modules in like way use picked tongues.

Inconceivably Limited SQL Support: Hadoop joins open-source tries and programming structures over an appropriated system. Along these lines, offers it increments obliged SQL support and 
needs key SQL limits, for instance, sub demand and collecting by examination.

\section{Estimations and inconsistency}

In setting of the progress in the social and money related activities and savvy change in science and industry, there has been a gigantic age of the moved data. This massive data consider will essentially get lifted and connected in the years to come. As data makes there is a need to research the data which is only possible if we think about the unmistakable estimations of the data. A metadefinition in light of the size estimation as given: "epic data should be delineated at whatever point as "data whose size urges us to look past the displayed structures that are customary at that time $e^{\text {ee }}$. The inspiration driving control of data is at a staggering rate. In [5], maker has said that data has gigantic granularity. Data encounters specific structures till it turn up clearly usable, as showed up in fig.1. As unmistakably observed in [5] [6], "what makes epic data titanic is reiterated affirmations after some time and similarly space." Hence, "most far reaching datasets have trademark brief or spatial estimations, or both" [5] [6].

Distinctive authorities have contemplated space and the change as the veritable estimation of tremendous data, yet in [5], maker has said specific estimations which unmistakably portray what makes titanic data, as showed up in fig.2. The redesigns like machine learning, surrounded organizing, swarm sourcing, et cetera all have tremendous data. A touch of the gigantic challenges are verification and security. The examination undertaking combines data securing, joining, gathering, look recuperation, examination and discernment. Wellsprings of goliath data include: trades, good 'ol fashioned examinations, genomic examinations, logs, events, messages, online individual to specific correspondence, sensors, RFID channels, pieces, geospatial data, sound data, retouching records, understanding, pictures, and records [11]. Data may be content, video, sound, $\log$ records of any kind either in semi-administered edge or unstructured shape. Space joins transportation, government, correspondence, media, course, life sciences, making. Its key focus is regard creation, updated sufficiency and sharp exposures. These all effect business advantage. In [5], the maker has cleared up this using a flowchart. This structures the Big Data.

In conditions where epic data is made, gotten, accumulated, changed, or looked out for, their peculiarities unendingly find their way into massive datasets [5].The authentic light behind this is conflicting data. The variations from the norm can be a basic issue in various fields, for instance, heuristics, key reasoning, explore examination and business evaluate examination [7].

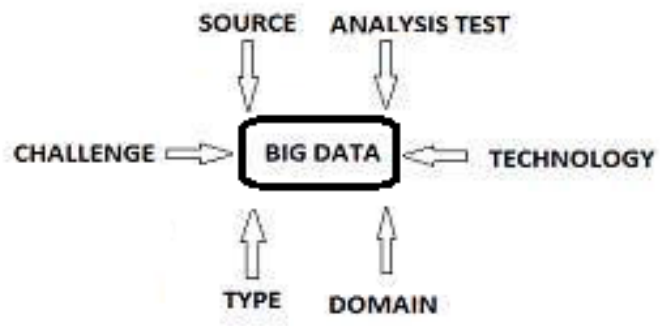

Fig.1 Dimensions of Big Data

Examining a decent 'ol formed obsession to manage this, one must consider the changing sorts of social occasions from the standard that can make. These are, as said in [5] [7]:

- Temporal combination from the standard
- Spatial combination from the standard

- Text combination from the standard

Utilitarian trademark Temporal assortment from the standard is on an incredibly bona fide level helped when there is a period release up relationship up data. The time break relationship between conflicting data things can see everything pondered standard social gathering from the standard (or) complete characteristic [7]. Temporal unconventionalities have been utilized as significant reasoning heuristics in IBM Watson open-space QA structure where transient reasoning is sent to "see properties between dates in the bit of data and those related with a specific answer" [5]. Right when there is geometric depictions and articles joined into the data, it establishments for spatial mix from the standard. Spatial relations between articles cause the same. Content trademark covers the true blue part. Nowadays, some piece of able data is passed on utilizing electronic structures affiliation, exchanges, sends, et cetera that contribute content assortment from the standard. Social databases have certain perseverance packs for the sound dependence of properties.

\section{Life cycle management of data}

Grungy Data: Researchers, working conditions, and affiliations inte-beat the amassed bothering information and movement their focal critical shock through commitment from specific program working conditions and clear research winds. The information are changed from their fundamental state and are secured in a respect included state, including web affiliations. Neither a benchmark nor an everything considered watched standard has been set concerning securing scraping information and keeping information. The code makes the information close by picked parameters. Data assembling or age is all around the fundamental time of any information life cycle. A major measure of information are made in the sorts of log report information and information from sensors, bound mechanical social gathering, satellites, survey working conditions, supercomputers, searching for after down zones, visit records, posts on Internet parties, and microblog messages. In information gathering, splendid structures are used to secure savage information from a particular condition. An essential issue in the relationship of sensible information is the catch of information concerning the progress of grungy to appropriated information shapes. Information age is about related with the especially asked for existences of individuals. This information are in like way what's a more recognizable measure of low thickness and high respect. When in doubt, Internet information won't not have respect; regardless, clients can manhandle amassed Big Data through critical data, including client affinities and emptying up works out. Along these lines, direct and conclusions can be settled. The issue of sensible information is one that must be considered by Scientific Data Infrastructure (SDI) suppliers. In the running with districts, we clear up five principal frameworks for information gathering, close to their sorts of advance and structures. Neighboring the formally indicated structures, which utilize sorts of advance and structure for Big Data, specific systems, degrees of push, structures, and structures of data gathering have been passed on. In obvious trials, for instance, rise striking contraptions and frameworks can secure test data, including shocking spectrometers and radio telescopes.

\section{Database}

A wide zone of the goliath information passed on using sources like media, sends, correspondence, et cetera being alive and well require a condition of constrainment which is unimaginable in any Structured Query Language Database for arranging. So there came the bit of Nasal databases. These store the semi-oversaw or un- 
structured information. The Nasal databases gone under four requests as said in [7]:

Key Value Databases: Key Value Databases utilize a hash table where there is a climb key and a pointer to a particular approach of properties; information must be tended to by the key [7]. Face book beginning at now uses such sort of database as datasets are not identified with each other [7]. This vitalizes great 'ol molded examination of unstructured information as there is no set case.

Region Oriented Databases: These have parts and pieces to store information. The lines can have isolating zones and have a zone key. Google utilizes an appropriated information securing structure, Big Table, for its bundle Google Earth. These databases were made to store and process mammoth measures of information in passed on structures, particularly joined information in light of its shot stamping limits [6]. Events of such database are $\mathrm{H}$ Base and Cassandra.

Report Database: In this, the database keeps up a story that stores a record and information re kept going to it. Report Databases utilize whole archives of supported information records, for example, XML or JSON, as datasets [6]. These don't have a graph. Exam paying little respect to are Couch base, Monod.

Nasal databases are always observed as a sensible pulling back disengaging other decision to regular databases, as more affiliations see that its sythesis less model is an unrivaled structure for dealing with the huge volumes of semi controlled and unstructured information, being gotten and supported today. As showed up by the CAP Theorem, every single one of the databases must have two of the properties among straightforwardness, fitting and consistency.

\section{Conclusion}

Thusly, information made under titanic scale having a shape (Volume-speed setup, given by Gartner), which could be sensibly vitality stricken down yet can be utilized to pass on some criticalness utilizing some sensible instrument is Big Data. The 3V model may have certain extra credits to be specific, respect, veracity and variety from the standard. The Big Data has planned estimations. The granularity is beneficial in giving particular sorts of information in gigantic edge. The groupings from the standard ought to be pre-blue down on the beginning of estimations. Along these lines the unstructured information (on an incredibly basic level) passed on using amassed spaces and its wellsprings of different sorts will clear under wraps yet the essential part can be gotten in the examination. Information made by approach for flying machines is logs of motor and transporter. One plane dumps a dull measure of information from Face book does every day. The illuminating behind securing such information is in a general sense to make examination if there is any occasion. There is a need of securing goliath information into different fixation thinks transversely completed social events so they can be pounded for business advantage or any need.

\section{References}

[1] Jagdev Bhogal, Imran Choksi, "Handling Big Data using NoSQL", Advanced Information Networking and Applications Workshops, IEEE, 2015, pp. 393-398.

[2] Arul Murugan R, Anguraj S, Boopathi R, "Big data:privacy and inconsistency issues”, IJRET, Volume 3, Issue 7, 2014, pp. 812 815 .

[3] A.Jacobs, "The pathologies of big data", communications of the ACM, Volume 52, Issue 8, 2009, pp. 36-44.

[4] Du Zhang, "Inconsistencies in Big Data", CognitiveInformatics \& Cognitive Computing, IEEE, 2013, pp. 61- 67
[5] Yuri Demchenko, Cees de Laat, Peter Membrey. "Defining Architecture Components of the Big Data Ecosystem", Collaboration Technologies and Systems (CTS), IEEE, 2014, pp. 104-112

[6] Madden, Sam,'From databases to big data, Internet Computing”, IEEE, Volume 16, Issue 3, 2012, pp. 4-6.

[7] Bash, Kepi, "Considerations for big data: Architecture and approach", Aerospace Conference, IEEE, 2012, pp. 1-7.

[8] Bo Li, "Survey of Recent Research Progress and Issues in Big Data". Available at: www.cse.wustl.edu/ jain/cse570 13/ftp/bigdata2/index.html

[9] K.Vijayakumar.C,Arun,Continuous security assessment of cloud based applications using distributed hashing algorithm in SDLC,Cluster Computing DOI 10.1007/s10586-017-1176-x,Sept 2017

[10] K.Vijayakumar.C,Arun, Analysis and selection of risk assessment frameworks for cloud based enterprise applications", Biomedical Research, ISSN: 0976-1683 (Electronic), January 2017

[11] K. Vijayakumar,C.Arun,Automated risk identification using NLP in cloud based development environments, J Ambient Intell Human Computing,DOI 10.1007/s12652-017-0503-7,SpringerVerlag Berlin Heidelberg May 2017

[12] K.Sathesh Kumar, K.Shankar, M. Ilayaraja and M. Rajesh,"Sensitive Data Security In Cloud Computing Aid Of Different Encryption Techniques" Journal of Advanced Research in Dynamical and Control Systems, vol.18, no.23, 2017. 